\title{
Antiferromagnetic spatial photonic Ising machine through optoelectronic correlation computing
}

\author{
Zhichao Ruan ( $\nabla$ zhichao@zju.edu.cn ) \\ Zhejiang University https://orcid.org/0000-0001-8311-6970 \\ Huang Junyi \\ Zhejiang University \\ Yisheng Fang \\ Zhejiang University
}

\section{Article}

Keywords: spatial photonic Ising machines (SPIM),optoelectronic correlation computing, condensedmatter physics

Posted Date: May 18th, 2021

DOl: https://doi.org/10.21203/rs.3.rs-478299/v1

License: (9) This work is licensed under a Creative Commons Attribution 4.0 International License. Read Full License

Version of Record: A version of this preprint was published at Communications Physics on November 12th, 2021. See the published version at https://doi.org/10.1038/s42005-021-00741-x. 


\title{
Antiferromagnetic spatial photonic Ising machine through optoelectronic correlation computing
}

\author{
Junyi Huang,* Yisheng Fang, ${ }^{*}$ and Zhichao Ruan ${ }^{\dagger}$ \\ Interdisciplinary Center of Quantum Information, \\ State Key Laboratory of Modern Optical Instrumentation, \\ and Zhejiang Province Key Laboratory of Quantum Technology and Device, \\ Department of Physics, Zhejiang University, Hangzhou 310027, China
}

\begin{abstract}
Recently, spatial photonic Ising machines (SPIM) have been demonstrated to compute the minima of Hamiltonians for large-scale spin systems. Here we propose to implement an antiferromagnetic model through optoelectronic correlation computing with SPIM. Also we exploit the gauge transformation which enables encoding the spins and the interaction strengths in a single phase-only spatial light modulator. With a simple setup, we experimentally show the ground state search of an antiferromagnetic model with 40000 spins in number-partitioning problem. Thus such an optoelectronic computing exhibits great programmability and scalability for the practical applications of studying statistical systems and combinatorial optimization problems.
\end{abstract}

\section{INTRODUCTION}

The spin glass models are widely used for investigations of interacting systems in both science and engineering $[1-12]$. In the past decades, the developments in spin machines have generated tremendous interest due to the prospects of solving a large class of NP-hard problems by searching the ground states of spin system Hamiltonians [13]. Optimization problem solvers with remarkable performance have been demonstrated in various systems, e.g. trapped ions [14-16], atomic and photonic condensates [17, 18], superconducting circuits [19], coupled parametric oscillators [20-30], injection-locked or degenerate cavity lasers [31-34], integrated nanophotonic circuits [3540], and polaritons [41-43].

Recently, like optical analog computations exploring the spatial degrees of freedom [44-52], the spatial photonic Ising machine (SPIM) has been proposed with reliable large-scale Ising spin systems, even up to thousands of spins [53]. With spatial light modulations, these spatial Ising spin setups benefit from the high speed and parallelism of optical signal processing [53-57]. Although the modeling of ferromagnetic and spin glass systems has been demonstrated [58], how to implement antiferromagnetic models in SPIM has not been proposed yet. In particular, the antiferromagnetic Ising models are important and extensive in research fields like oxide materials [59] and giant magnetoresistance [60, 61]. Also, the combinatorial optimization problems with antiferromagnetic Ising models have many real-world applications such as multiprocessor scheduling, minimization of circuit size and delay, cryptography, and logistics analysis $[62,63]$.

In this Work, we propose to implement the antiferromagnetic model through optoelectronic correlation computing. We show that an antiferromagnetic Hamilto-

\footnotetext{
* These authors contributed equally to this work.

† zhichao@zju.edu.cn
}

nian can be evaluated through the correlation between a distribution function and the measured optical intensity with SPIM. We experimentally demonstrate the groundstate-search process with a number-partitioning problem, where 40000 spins are connected with random antiferromagnetic interaction strengths. Our results show that the proposed antiferromagnetic model in SPIM can evolve toward the ground state, exhibiting an efficient approach by scalable degrees of freedom in spatial light modulation.

\section{RESULTS}

\section{A. Optoelectronic correlation computing for Mattis-type Ising model}

We first introduce the gauge transformation for SPIM, which encodes the spins and the interaction strengths in a single phase-only spatial light modulator (SLM) [58]. We consider a Mattis-type spin glass system with the Ising model Hamiltonian $H=-\sum_{j h} J_{j h} \sigma_{j} \sigma_{h}$, where the spin configuration is $\mathbf{S}=\left\{\sigma_{j}\right\}(j=1,2, \cdots N)$ and $\sigma_{j}$ takes binary value of either +1 or -1 , representing the spin-up or spin-down state respectively. The interaction strengths can be expressed as $J_{j h}=J \xi_{j} \xi_{h}$, where $J$ is an interaction strength as a function of the distance between two spins with the unit of energy, and the amplitude modulation $\xi_{j}$ is limited as $-1 \leq \xi_{j} \leq 1$. By the gauge transformation shown in Fig. 1(a), when rotating each original spin $\sigma_{j}$ with the angle $\alpha_{j}=\arccos \xi_{j}$, the new spin vector $\sigma_{j}^{\prime}$ is projected on the $z$-axis to obtain the effective spin $\sigma_{j}^{\prime z}=\xi_{j} \sigma_{j}$. As a result, the gauge transformation keeps the Hamiltonian invariant $H=-\sum_{j h} J{\sigma_{j}^{\prime}}^{z}{\sigma_{h}^{\prime}}^{z}$, while the interactions between the $z$ components of gauge-transformed spins are equal to the strength $J$.

The gauge invariance property promises that the experimental implementation only needs a single phase- 
(a)

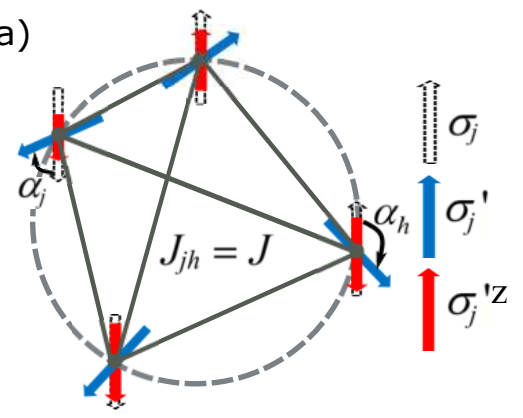

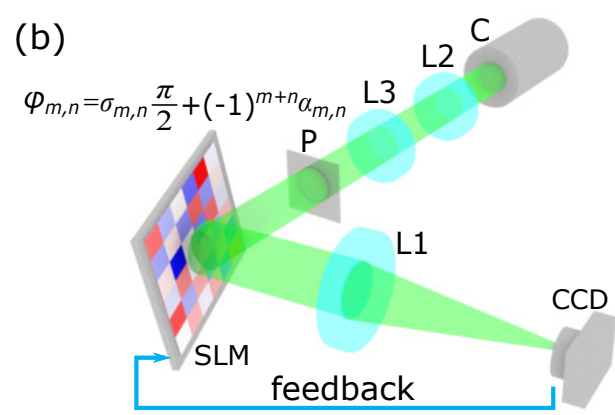

FIG. 1. (a) Schematic of the gauge transformation. The dotted, blue and red arrow correspond to the origin spin $\sigma_{j}$, the new spin vector $\sigma_{j}^{\prime}$ and the effective spin vector $\sigma_{j}^{\prime}{ }^{z}$, respectively. (b) The experimental optical setups for SPIM: SLM, spatial light modulator; C, collimator; L, lens; P, polarizer. A collimated laser beam illuminates the SLM. By gauge transformation, the gauge-transformed effective spin configuration $\mathbf{S}^{\prime z}=\left\{{\sigma_{m, n}^{\prime}}^{z}\right\}$ is encoded through a single phase-only SLM following Eq. (1). The modulated light is detected by a CCD camera in the back-focus plane of Lens L1.

only SLM, with uniform illumination by a collimated uniform laser beam [Fig. 1(b)]. This setup circumvents the difficulty of pixel alignment in the previously proposed SPIM [53-56], and therefore greatly improves the system stability and the computing fidelity. Since the spins are loaded through two-dimensional spatial modulation, the $j$-th spin is distributed in a square lattice at $\mathbf{j}=(m, n)$, where $1 \leq m \leq N_{x}, 1 \leq n \leq N_{y}$. According to Ref.[58], after the gauge transformation, each spin is encoded by a macropixel with phase modulation $\varphi_{m, n}$ such that $\sigma_{j}^{\prime z}=\exp \left(i \varphi_{m, n}\right)$ and

$$
\varphi_{m, n}=\sigma_{m, n} \frac{\pi}{2}+(-1)^{m+n} \alpha_{m, n}
$$

Then with Lens L1 of the focal length $f$ performing Fourier transformation, we detect the band-limited intensity distribution $I(\mathbf{u})$ confined within the first diffraction order zone $A$ on the focal plane, and $I(\mathbf{u})=$ $\sum_{j h}{\sigma_{j}^{\prime}}^{z} \sigma_{h}^{\prime z} e^{i \frac{2 \pi}{f \lambda}\left(\mathbf{x}_{j}-\mathbf{x}_{h}\right) \cdot \mathbf{u}} \operatorname{sinc}^{2}\left(\frac{\mathbf{u} W}{f \lambda}\right)$, where $\lambda$ is the wavelength, $f$ is the focal length of lens L1, $W$ is the length of each macropixel, $\mathbf{x}_{j}=W \mathbf{j}$ is the center position of the $j$-th pixel, $\mathbf{u}=(u, v)$ is the spatial coordinate in the focal plane, and $\operatorname{sinc}(\mathbf{u})=\frac{\sin \pi u}{\pi u} \frac{\sin \pi v}{\pi v}$. Suppose that we preset a distribution function $g_{c}(\mathbf{u})$ and evaluate the correlation function $F$ as

$$
F=\int_{A} I(\mathbf{u}) g_{c}(\mathbf{u}) \mathrm{d} \mathbf{u}=\sum_{j h} G(\mathbf{j}-\mathbf{h}){\sigma_{j}^{\prime}}^{z}{\sigma_{h}^{\prime}}^{z} .
$$

Here

$$
G(\mathbf{k})=\int_{A} g_{c}(\mathbf{u}) \operatorname{sinc}^{2}\left(\frac{W \mathbf{u}}{f \lambda}\right) e^{i \frac{2 \pi W}{f \lambda} \mathbf{k} \cdot \mathbf{u}} \mathrm{d} \mathbf{u},
$$

that is, $G(\mathbf{k})$ is the Fourier transformation of $g_{c}(\mathbf{u}) \operatorname{sinc}^{2}\left(\frac{W \mathbf{u}}{f \lambda}\right)$. Indeed, Eq. (2) shows that by presetting an appropriate $g_{c}$, a Mattis-type Ising Hamiltonian can be evaluated as

$$
H=-F=-\sum_{j h} G(\mathbf{j}-\mathbf{h}) \sigma_{j}^{\prime z} \sigma_{h}^{\prime z} .
$$

We note that the distribution function $g_{c}(\mathbf{u})$ is distinct from the target intensity $I_{T}(\mathbf{u})$ proposed in Ref. [53]. Here $g_{c}(\mathbf{u})$ can be an arbitrary real function to guarantee an even function of the interaction strength [c.f. Eq. (3)], which has either positive or negative values, while the target intensity $I_{T}$ always has non-negative values. In particular, for the antiferromagnetic model, all the interaction strengths $J_{j h}<0$. In the case that $\xi_{j}$ s are positive, $G(\mathbf{j}-\mathbf{h})$ should be negative to ensure antiferromagnetic interactions between all the spins. It leads that $g_{c}$ must be negative for some values of $\mathbf{u}$, otherwise Eq. (3) shows $G>0$ for $\mathbf{j}=\mathbf{h}$. Moreover, when $g_{c}$ has both positive and negative values, the Hamiltonian can be evaluated through the correlation function as Eq. (2), while it cannot be implemented by the target-intensity approach.

\section{B. Number-partitioning problem with the antiferromagnetic Hamiltonian}

The antiferromagnetic model in SPIM provides a new computation platform for studying the challenging combinatorial optimization problems. As a demonstration, here we present the ground-state-search process of a combinatorial optimization problem, the NP-hard numberpartitioning problem [13, 64]: One would like to divide a set $\Xi=\left\{\xi_{j}\right\}$, containing $N$ real numbers $(j=1,2, \cdots N)$, into two subsets $\Xi_{1}$ and $\Xi_{2}$, such that the difference between the summations of elements in two subsets $\sum_{1}=$ $\sum_{\xi_{j} \in \Xi_{1}} \xi_{j}$ and $\sum_{2}=\sum_{\xi_{j} \in \Xi_{2}} \xi_{j}$ is as small as possible. Without loss of generality, we suppose all $\xi_{j}$ s in the set $\Xi$ are real numbers belonging to the range $(0,1]$. Specifically, when the number set $\Xi$ has parity symmetry, the spins of such models can be analytically optimized. For instance, for the set with an even total number of $\xi_{j} \mathrm{~s}$, when $\xi_{j}=\xi_{N+1-j}$, the spin should be $\sigma_{j}=-\sigma_{N+1-j}$ to ensure the equivalence of two subsets. In general, by labeling the elements belonging to two different subsets $\Xi_{1}$ and $\Xi_{2}$ with $\sigma_{j}=1$ and -1 respectively, the optimization 
is equivalent to minimizing the antiferromagnetic Hamiltonian $H=\left(\sum_{j} \xi_{j} \sigma_{j}\right)^{2}=\sum_{j h} \xi_{j} \xi_{h} \sigma_{j} \sigma_{h}$.

To implement such an antiferromagnetic Hamiltonian in SPIM, we explore the gauge transformation to search a spin configuration $\mathbf{S}^{\prime}=\left\{{\sigma_{j}^{\prime}}^{z}\right\}$ where ${\sigma_{j}^{\prime}}^{z}=\xi_{j} \sigma_{j}$ while keeping the interaction strength between any two spins $G(\mathbf{k})=-1$. Due to the gauge invariance, the Hamiltonian is $H=\sum_{j h} \sigma_{j}^{\prime z} \sigma_{h}^{\prime z}$ and the optimized value of $\left|\sum_{1}-\sum_{2}\right|$ is the total magnetization strength of the gauge-transformed spins $\left|m^{\prime}\right|=\frac{1}{N}\left|\sum_{j} \sigma_{j}^{\prime z}\right|$. During the experimental iterations, the spin configuration is updated gradually [53], and the system definitely evolves to the ground states, indicating the process of solving the optimization problem.

\section{Experimental ground state search}

We experimentally demonstrate the ground-statesearch process with the number-partitioning problem. As shown in Fig. 1(b), a collimated Gaussian beam (wavelength $\lambda=532 \mathrm{~nm}$ ) is expanded by two confocal lenses L2 (50mm focal length) and L3 (500mm focal length). After expansion, the waist radius of the collimated beam is about $36 \mathrm{~mm}$. Then a polarizer $\mathrm{P}$ is used to prepare the incident beam linearly polarized along the long display axis of the SLM (Holoeye PLUTO-NIR-011). The SLM is calibrated through the two-shot method based on generalized spatial differentiator [65]. Lens L1 with the focal length $f=100 \mathrm{~mm}$ performs Fourier transformation, where a CCD beam profiler (Ophir SP620) is used to detect the optical field intensity on the back focal plane.

As an example, we demonstrate the searching process with the number-partitioning problem of a set $\Xi=\left\{\xi_{j}\right\}$ with $N=40000$ elements. The set $\Xi$ is randomly generated that each element $\xi_{j}$ is a real number randomly chosen from $(0,1]$, as presented in Fig. 3(a) in the form of a 200-by-200 array. Here each spin is encoded by a macropixel with 2-by-2 pixels on SLM with the length of $W=16 \mu \mathrm{m}$. Since the beam size is much larger than that of the array, we assume that light illuminates each macropixel with a uniform amplitude.

In order to evaluate the correlation function $F$, we first numerically calculate the distribution function $g_{c}(\mathbf{u})$ through Eq. (3). Given the interaction strength between any two spins $G(\mathbf{k})=-1$, Fig. 2 (a) shows the calculated distribution function $g_{c}(\mathbf{u})$. We note that $g_{c}(\mathbf{u})$ have both positive and negative values, which means such a case cannot be implemented by the target-intensity approach as Ref. [53]. We also need to calibrate the intensity measurement such that the distribution function $g_{c}(\mathbf{u})$ has the same origin as the intensity distribution $I(\mathbf{u})$. In order to reduce the impact of optical aberrations, we measure the intensity distribution on $\mathrm{CCD}$
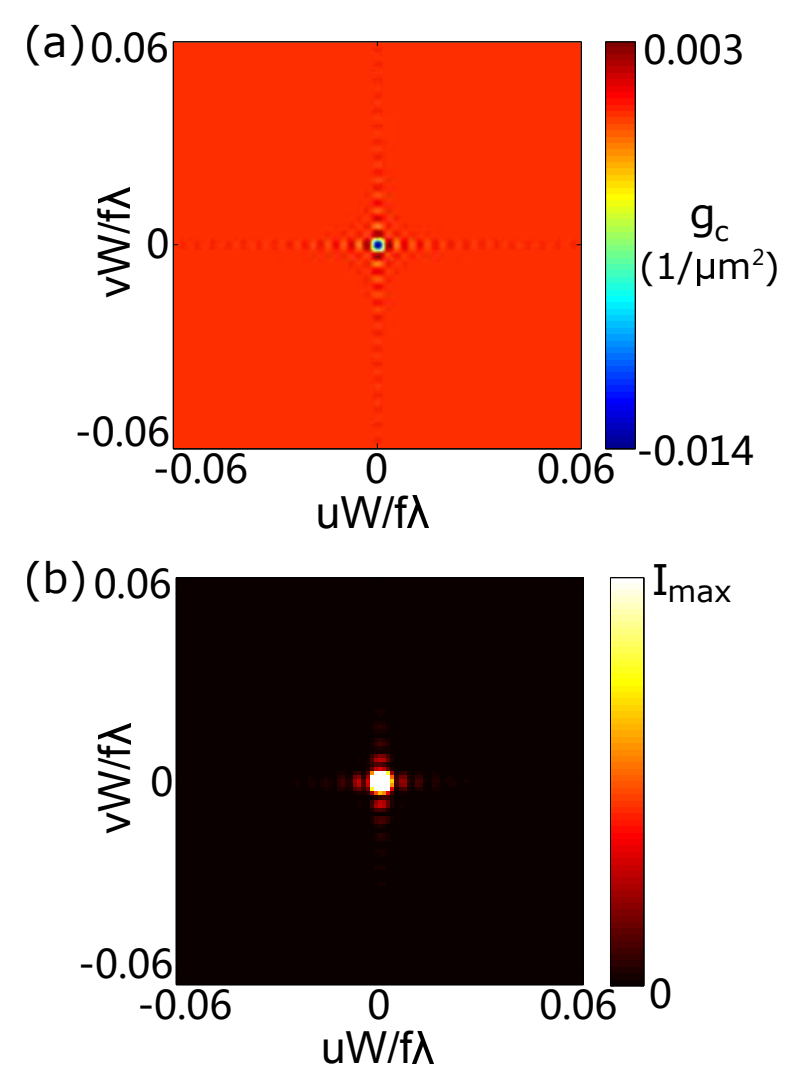

FIG. 2. (a) Calculated distribution function $g_{c}(\mathbf{u})$ of the antiferromagnetic Mattis model, where the interaction strength between any two spins $G(\mathbf{k})=-1$. (b) By setting the SLM with uniform phase modulation, we measure the intensity on CCD plane and thus determine the origin of coordinates at the maximal intensity.

plane [Fig. 2(b)] by setting the SLM with uniform phase modulation. Therefore, the origin of $I(\mathbf{u})$ is marked at the maximal intensity through the numerical fitting.

Next we start with the initial state that all spins are uniformly distributed as $\sigma_{j}=1$ and update the spin configuration for ground state search. Here we utilize the Markov chain Monte Carlo algorithm, where $\sigma_{j}$ s are tentatively updated during each iteration, and the gaugetransformed spins $\sigma_{j}^{\prime z}=\xi_{j} \sigma_{j}$ are encoded on SLM following Eq. (1). Then we measure the intensity $I(\mathbf{u})$ on the CCD and evaluate the system Hamiltonian through the correlation function $F$ as Eq. (2). The updated spin configuration is accepted only when the Hamiltonian decreases.

Figure 3(b) shows the evolution of the Hamiltonian $H$ and the amplitude of the gauge-transformed magnetization $\left|m^{\prime}\right|$ during the ground-state-search process. In the experiment, four independent trials are conducted with the initial state that all spins are uniformly distributed. For all the four cases, the Hamiltonian $H$ and the magnetization $\left|m^{\prime}\right|$ decrease rapidly at the beginning of the iteration, because the initial spin configuration strongly deviates from the ground state. As the number of iterations increases, the Hamiltonian tends to be stable, 
(a)

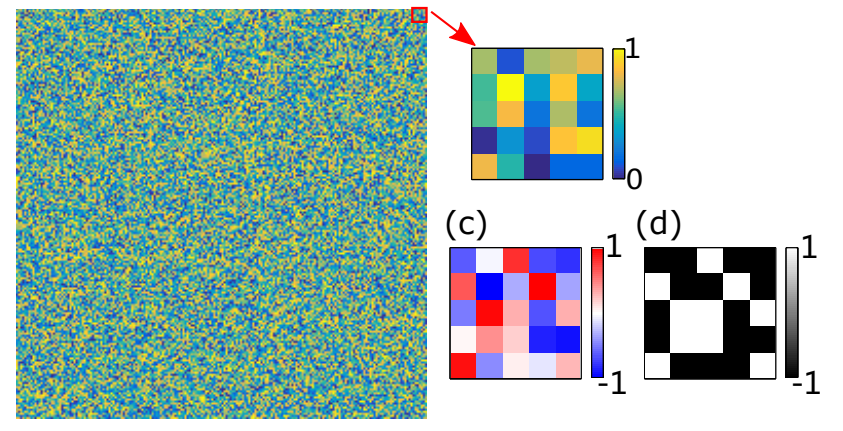

(b)

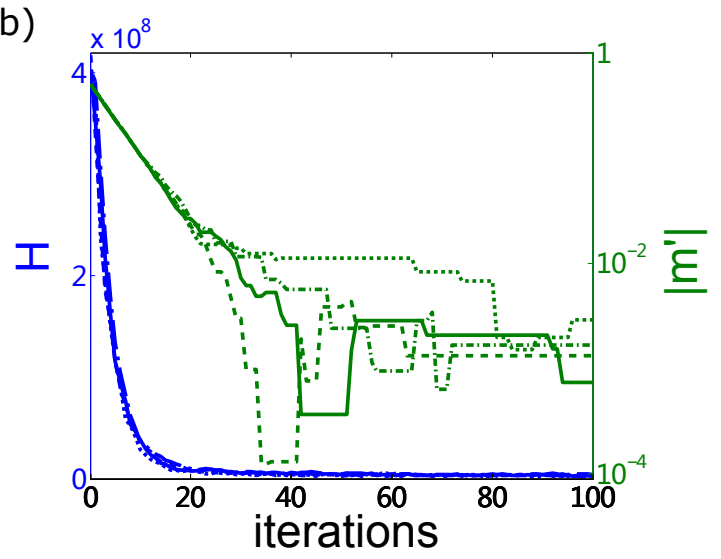

FIG. 3. Experimental results for the searching process of number-partitioning problem. (a) The set $\Xi=\left\{\xi_{j}\right\}$ containing 40000 elements, where each $\xi_{j}$ is chosen randomly from $(0,1]$ with uniform probability. (b) Evolutions of the Hamiltonian $H$ and amplitude of the gauge-transformed magnetization $\left|m^{\prime}\right|$ during the iterations. Four independent trials are conducted with uniform initial spin configurations. For clear visualization, (c) and (d) respectively presents only a part of the final configurations of the gauge-transformed spins $\left\{{\sigma_{j}^{\prime}}^{z}\right\}$ on SLM and the original spins $\left\{\sigma_{j}\right\}$, which correspond to the red square box in (a).

while the $\left|m^{\prime}\right|$ starts to fluctuate. We attribute it to the too weak intensity distribution $I(\mathbf{u})$, which is strongly affected by the noise during the measurement. As a result, the spin configuration may be incorrectly updated with the distribution resulting in a larger $\left|m^{\prime}\right|$. The accuracy can be improved by using a wide dynamic-range detector for intensity measurement, or by adjusting the input light intensity in real time within a suitable range.

Here for clear visualization, corresponding to the red square in Fig. 3(a), Figs. 3(c) and (d) present a part of the final configurations of the gauge-transformed spins $\mathbf{S}^{\prime}=\left\{\sigma_{j}^{\prime z}\right\}$ and the resulting spin configuration $\left\{\sigma_{j}\right\}$, respectively. Overall, for all these four trials, $\left|m^{\prime}\right|$ reaches lower than $1.7 \times 10^{-3}$ within 100 iterations. Thus during the ground state search, the magnetization $\left|m^{\prime}\right|$ decreases by nearly three orders of magnitude, which indicates the validity of the gauge transformation method for antiferromagnetic model.

\section{CONCLUSION AND DISCUSSION}

We propose to implement antiferromagnetic model in SPIM. By gauge transformation, an antiferromagnetic Hamiltonian can be evaluated through the correlation between the distribution function and the measured optical intensity with SPIM. To improve the processing speed of the system, the ultrafast SLM and CCD at gigahertz rates with the most recent technologies $[66,67]$ is helpful and practical. Also, the computing accuracy can be im- proved with a more sensitive CCD camera. We note that our proposed method can be applied to the ground-statesearch process, e.g., adiabatic evolution and simulated annealing algorithms $[68,69]$.

In summary, we optically demonstrate the groundstate-search process of an antiferromagnetic Mattis model with thousands of spins, as well as the numberpartitioning problem. With the improved accuracy resulting from gauge transformation, we successfully reduce the total magnetization strength of the gaugetransformed spins $\left|m^{\prime}\right|$ by nearly three orders of magnitude. Thus for practical applications in modeling statistical systems and studying combinatorial optimization problems, such an optoelectronic computing exhibits great programmability and scalability in large-scale systems.

\section{ACKNOWLEDGEMENT}

The authors acknowledge funding through the National Natural Science Foundation of China (NSFC Grants Nos. 91850108 and 61675179), the National Key Research and Development Program of China (Grant No. 2017YFA0205700), the Open Foundation of the State Key Laboratory of Modern Optical Instrumentation, and the Open Research Program of Key Laboratory of 3D Micro/Nano Fabrication and Characterization of Zhejiang Province.

The authors declare no conflict of interest.
[1] K. Binder and A. P. Young, Spin glasses: Experimental facts, theoretical concepts, and open questions, Reviews of Modern Physics 58, 801 (1986).

[2] S. F. Edwards and P. W. Anderson, Theory of spin 
glasses, Journal of Physics F: Metal Physics 5, 965 (1975).

[3] D. Sherrington and S. Kirkpatrick, Solvable model of a spin-glass, Physical Review Letters 35, 1792 (1975).

[4] M. Gabay and G. Toulouse, Coexistence of spin-glass and ferromagnetic orderings, Physical Review Letters 47, 201 (1981).

[5] J. D. Reger and A. Zippelius, Three-dimensional randombond Ising model: Phase diagram and critical properties, Physical Review Letters 57, 3225 (1986).

[6] G. De las Cuevas, W. Dur, H. J. Briegel, and M. A. Martin-Delgado, Unifying all classical spin models in a lattice gauge theory, Physical Review Letters 102, 230502 (2009).

[7] M. C. Bañuls, R. Blatt, J. Catani, A. Celi, J. I. Cirac, M. Dalmonte, L. Fallani, K. Jansen, M. Lewenstein, S. Montangero, et al., Simulating lattice gauge theories within quantum technologies, The European Physical Journal D 74, 1 (2020).

[8] J. J. Hopfield, Neural networks and physical systems with emergent collective computational abilities, Proceedings of the National Academy of Sciences 79, 2554 (1982).

[9] D. J. Amit, H. Gutfreund, and H. Sompolinsky, Spinglass models of neural networks, Physical Review A 32, 1007 (1985).

[10] E. Agliari, A. Barra, A. De Antoni, and A. Galluzzi, Parallel retrieval of correlated patterns: From Hopfield networks to Boltzmann machines, Neural Networks 38, 52 (2013).

[11] N. Sourlas, Spin-glass models as error-correcting codes, Nature 339, 693 (1989).

[12] H. Nishimori, Statistical physics of spin glasses and information processing: an introduction, 111 (Clarendon Press, 2001).

[13] A. Lucas, Ising formulations of many NP problems, Frontiers in Physics 2, 5 (2014).

[14] K. Kim, M.-S. Chang, S. Korenblit, R. Islam, E. E. Edwards, J. K. Freericks, G.-D. Lin, L.-M. Duan, and C. Monroe, Quantum simulation of frustrated Ising spins with trapped ions, Nature 465, 590 (2010).

[15] J. W. Britton, B. C. Sawyer, A. C. Keith, C.-C. J. Wang, J. K. Freericks, H. Uys, M. J. Biercuk, and J. J. Bollinger, Engineered two-dimensional ising interactions in a trapped-ion quantum simulator with hundreds of spins, Nature 484, 489 (2012).

[16] X.-s. Ma, B. Dakic, W. Naylor, A. Zeilinger, and P. Walther, Quantum simulation of the wavefunction to probe frustrated heisenberg spin systems, Nature Physics 7, 399 (2011).

[17] J. Struck, M. Weinberg, C. Ölschläger, P. Windpassinger, J. Simonet, K. Sengstock, R. Höppner, P. Hauke, A. Eckardt, M. Lewenstein, et al., Engineering Ising-XY spin-models in a triangular lattice using tunable artificial gauge fields, Nature Physics 9, 738 (2013).

[18] B. Kassenberg, M. Vretenar, S. Bissesar, and J. Klaers, Controllable Josephson junction for photon BoseEinstein condensates, arXiv preprint arXiv:2001.09828 (2020).

[19] M. W. Johnson, M. H. S. Amin, S. Gildert, T. Lanting, F. Hamze, N. Dickson, R. Harris, A. J. Berkley, J. Johansson, P. Bunyk, et al., Quantum annealing with manufactured spins, Nature 473, 194 (2011).

[20] P. L. McMahon, A. Marandi, Y. Haribara, R. Hamerly,
C. Langrock, S. Tamate, T. Inagaki, H. Takesue, S. Utsunomiya, K. Aihara, et al., A fully programmable 100spin coherent Ising machine with all-to-all connections, Science 354, 614 (2016).

[21] T. Inagaki, Y. Haribara, K. Igarashi, T. Sonobe, S. Tamate, T. Honjo, A. Marandi, P. L. McMahon, T. Umeki, K. Enbutsu, et al., A coherent Ising machine for 2000node optimization problems, Science 354, 603 (2016).

[22] T. Inagaki, K. Inaba, R. Hamerly, K. Inoue, Y. Yamamoto, and H. Takesue, Large-scale Ising spin network based on degenerate optical parametric oscillators, Nature Photonics 10, 415 (2016).

[23] F. Böhm, G. Verschaffelt, and G. Van der Sande, A poor man's coherent Ising machine based on opto-electronic feedback systems for solving optimization problems, Nature Communications 10, 3538 (2019).

[24] R. Hamerly, T. Inagaki, P. L. McMahon, D. Venturelli, A. Marandi, T. Onodera, E. Ng, C. Langrock, K. Inaba, T. Honjo, et al., Experimental investigation of performance differences between coherent Ising machines and a quantum annealer, Science Advances 5, eaau0823 (2019).

[25] L. Bello, M. Calvanese Strinati, E. G. Dalla Torre, and A. Pe'er, Persistent coherent beating in coupled parametric oscillators, Physical Review Letters 123, 083901 (2019).

[26] F. Böhm, T. Inagaki, K. Inaba, T. Honjo, K. Enbutsu, T. Umeki, R. Kasahara, and H. Takesue, Understanding dynamics of coherent Ising machines through simulation of large-scale 2D Ising models, Nature Communications 9, 5020 (2018).

[27] E. S. Tiunov, A. E. Ulanov, and A. Lvovsky, Annealing by simulating the coherent Ising machine, Optics Express 27, 10288 (2019).

[28] Z. Wang, A. Marandi, K. Wen, R. L. Byer, and Y. Yamamoto, Coherent Ising machine based on degenerate optical parametric oscillators, Physical Review A 88, 063853 (2013).

[29] Y. Yamamoto, K. Aihara, T. Leleu, K.-i. Kawarabayashi, S. Kako, M. Fejer, K. Inoue, and H. Takesue, Coherent Ising machines optical neural networks operating at the quantum limit, npj Quantum Information 3, 1 (2017).

[30] A. Marandi, Z. Wang, K. Takata, R. L. Byer, and Y. Yamamoto, Network of time-multiplexed optical parametric oscillators as a coherent Ising machine, Nature Photonics 8, 937 (2014).

[31] S. Utsunomiya, K. Takata, and Y. Yamamoto, Mapping of Ising models onto injection-locked laser systems, Optics Express 19, 18091 (2011).

[32] K. Takata, S. Utsunomiya, and Y. Yamamoto, Transient time of an Ising machine based on injection-locked laser network, New Journal of Physics 14, 013052 (2012).

[33] M. Babaeian, D. T. Nguyen, V. Demir, M. Akbulut, P.A. Blanche, Y. Kaneda, S. Guha, M. A. Neifeld, and N. Peyghambarian, A single shot coherent Ising machine based on a network of injection-locked multicore fiber lasers, Nature Communications 10, 3516 (2019).

[34] C. Tradonsky, I. Gershenzon, V. Pal, R. Chriki, A. A. Friesem, O. Raz, and N. Davidson, Rapid laser solver for the phase retrieval problem, Science Advances 5, eaax4530 (2019).

[35] C. Roques-Carmes, Y. Shen, C. Zanoci, M. Prabhu, F. Atieh, L. Jing, T. Dubček, C. Mao, M. R. Johnson, V. Ceperić, et al., Heuristic recurrent algorithms for photonic Ising machines, Nature Communications 11, 249 
(2020).

[36] M. Prabhu, C. Roques-Carmes, Y. Shen, N. Harris, L. Jing, J. Carolan, R. Hamerly, T. Baehr-Jones, M. Hochberg, V. Čeperić, et al., Accelerating recurrent Ising machines in photonic integrated circuits, Optica 7, $551(2020)$.

[37] Y. Shen, N. C. Harris, S. Skirlo, M. Prabhu, T. BaehrJones, M. Hochberg, X. Sun, S. Zhao, H. Larochelle, D. Englund, et al., Deep learning with coherent nanophotonic circuits, Nature Photonics 11, 441 (2017).

[38] K. Wu, J. G. De Abajo, C. Soci, P. P. Shum, and N. I. Zheludev, An optical fiber network oracle for NPcomplete problems, Light: Science \& Applications 3, e147 (2014).

[39] M. R. Vázquez, V. Bharadwaj, B. Sotillo, S.-Z. A. Lo, R. Ramponi, N. I. Zheludev, G. Lanzani, S. M. Eaton, and C. Soci, Optical NP problem solver on laser-written waveguide platform, Optics Express 26, 702 (2018).

[40] Y. Okawachi, M. Yu, J. K. Jang, X. Ji, Y. Zhao, B. Y. Kim, M. Lipson, and A. L. Gaeta, Nanophotonic spinglass for realization of a coherent ising machine, arXiv preprint arXiv:2003.11583 (2020).

[41] N. G. Berloff, M. Silva, K. Kalinin, A. Askitopoulos, J. D. Töpfer, P. Cilibrizzi, W. Langbein, and P. G. Lagoudakis, Realizing the classical XY Hamiltonian in polariton simulators, Nature Materials 16, 1120 (2017).

[42] K. P. Kalinin and N. G. Berloff, Simulating Ising and n-state planar potts models and external fields with nonequilibrium condensates, Physical Review Letters 121, 235302 (2018).

[43] K. P. Kalinin, A. Amo, J. Bloch, and N. G. Berloff, Polaritonic XY-Ising machine, Nanophotonics 9, 4127 (2020).

[44] A. Silva, F. Monticone, G. Castaldi, V. Galdi, A. Alù, and N. Engheta, Performing mathematical operations with metamaterials, Science 343, 160 (2014).

[45] D. A. Bykov, L. L. Doskolovich, E. A. Bezus, and V. A. Soifer, Optical computation of the laplace operator using phase-shifted bragg grating, Optics Express 22, 25084 (2014).

[46] Z. Ruan, Spatial mode control of surface plasmon polariton excitation with gain medium: from spatial differentiator to integrator, Optics Letters 40, 601 (2015).

[47] A. Youssefi, F. Zangeneh-Nejad, S. Abdollahramezani, and A. Khavasi, Analog computing by brewster effect, Optics Letters 41, 3467 (2016).

[48] T. Zhu, Y. Zhou, Y. Lou, H. Ye, M. Qiu, Z. Ruan, and S. Fan, Plasmonic computing of spatial differentiation, Nature Communications 8, 15391 (2017).

[49] W. Zhang, K. Cheng, C. Wu, Y. Wang, H. Li, and $\mathrm{X}$. Zhang, Implementing quantum search algorithm with metamaterials, Advanced Materials 30, 1703986 (2018).

[50] C. Guo, M. Xiao, M. Minkov, Y. Shi, and S. Fan, Photonic crystal slab laplace operator for image differentiation, Optica 5, 251 (2018).

[51] T. Zhu, Y. Lou, Y. Zhou, J. Zhang, J. Huang, Y. Li, H. Luo, S. Wen, S. Zhu, Q. Gong, et al., Generalized spatial differentiation from the spin hall effect of light and its application in image processing of edge detection, Physical Review Applied 11, 034043 (2019).

[52] F. Zangeneh-Nejad, D. L. Sounas, A. Alù, and R. Fleury,
Analogue computing with metamaterials, Nature Reviews Materials 6, 207 (2020).

[53] D. Pierangeli, G. Marcucci, and C. Conti, Large-scale photonic Ising machine by spatial light modulation, Physical Review Letters 122, 213902 (2019).

[54] D. Pierangeli, G. Marcucci, D. Brunner, and C. Conti, Noise-enhanced spatial-photonic Ising machine, Nanophotonics 9, 4109 (2020).

[55] D. Pierangeli, G. Marcucci, and C. Conti, Adiabatic evolution on a spatial-photonic Ising machine, Optica 7, 1535 (2020).

[56] D. Pierangeli, M. Rafayelyan, C. Conti, and S. Gigan, Scalable spin-glass optical simulator, Physical Review Applied 15, 034087 (2021).

[57] S. Kumar, H. Zhang, and Y.-P. Huang, Large-scale Ising emulation with four body interaction and all-to-all connections, Communications Physics 3, 1 (2020).

[58] Y. Fang, J. Huang, and Z. Ruan, Experimental observation of phase transitions in spatial photonic Ising machine (2021), arXiv:2011.02771 [cond-mat.dis-nn].

[59] C. G. Shull, W. Strauser, and E. Wollan, Neutron diffraction by paramagnetic and antiferromagnetic substances, Physical Review 83, 333 (1951).

[60] G. Binasch, P. Grünberg, F. Saurenbach, and W. Zinn, Enhanced magnetoresistance in layered magnetic structures with antiferromagnetic interlayer exchange, Physical Review B 39, 4828 (1989).

[61] M. N. Baibich, J. M. Broto, A. Fert, F. N. Van Dau, F. Petroff, P. Etienne, G. Creuzet, A. Friederich, and J. Chazelas, Giant magnetoresistance of (001) fe/(001) cr magnetic superlattices, Physical Review Letters 61, 2472 (1988).

[62] T. Graß, D. Raventós, B. Juliá-Díaz, C. Gogolin, and M. Lewenstein, Quantum annealing for the numberpartitioning problem using a tunable spin glass of ions, Nature Communications 7, 11524 (2016).

[63] S. Mertens, The easiest hard problem: Number partitioning, Computational Complexity and Statistical Physics 125, 125 (2006).

[64] S. Mertens, Phase transition in the number partitioning problem, Physical Review Letters 81, 4281 (1998).

[65] J. Huang, T. Zhu, and Z. Ruan, Two-shot calibration method for phase-only spatial light modulators with generalized spatial differentiator, Physical Review Applied 14, 054040 (2020).

[66] Q. Chen, S. Song, H. Wang, L. Liang, Y. Dong, and L. Wen, Ultra-broadband spatial light modulation with dual-resonance coupled epsilon-near-zero materials, Nano Research , 1 (2020).

[67] J. Park, B. G. Jeong, S. I. Kim, D. Lee, J. Kim, C. Shin, C. B. Lee, T. Otsuka, J. Kyoung, S. Kim, et al., All-solidstate spatial light modulator with independent phase and amplitude control for three-dimensional lidar applications, Nature Nanotechnology 16, 69 (2021).

[68] E. Farhi, J. Goldstone, S. Gutmann, J. Lapan, A. Lundgren, and D. Preda, A quantum adiabatic evolution algorithm applied to random instances of an np-complete problem, Science 292, 472 (2001).

[69] S. Kirkpatrick, C. D. Gelatt, and M. P. Vecchi, Optimization by simulated annealing, Science 220, 671 (1983). 